\title{
Mitigating switching variability in carbon nanotube memristors
}

\author{
J. Farmer, W. Whitehead, A. Hall, D. Veksler, G. Bersuker, ${ }^{1}$ \\ D. Gao, Al-Moatasem El-Sayed, T. Durrant, A. Shluger, 2,3 \\ T. Rueckes, L. Cleveland, H. Luan, R. Sen ${ }^{4}$ \\ 1. The Aerospace Corporation, El Segundo, CA, 90245, USA \\ 2. Nanolayers Research Computing LTD, London, N12 0HL, UK \\ 3. Department of Physics and Astronomy, University College London, London WC1E 6BT, UK \\ 4. Nantero Inc., Woburn, MA 01801, USA
}

\section{INTRODUCTION}

Carbon nanotube (CNT) relay devices represent promising class of resistive memory technologies (RRAM) where a current through the CNT film is modulated by changing contacts between connected nanotubes. A CNT memory cell (Fig. 1 (a)) consists of TiN electrodes sandwiching a 10 30 nm layer of CNT fabric formed by layers of randomly oriented nanotubes [1]. In such disordered network, neighboring individual CNTs can bound to each other via van der Waals forces. Resistance of a CNT network can vary within a wide range $\sim(1-100) \mathrm{M} \Omega$ depending on applied programming signals. Stochastic nature of the conductance update process presents challenges for CNT memristors implementation in neural network hardware, in particular, due to variability of resistance changes under identical programming operations, collapse of the memory updates (Fig. 2), etc.

In order to determine optimum operation conditions, we focus on identifying root-cause of instability. We consider that atomiclevel structural changes at specific sites are driven by local temperature affected by released energy generated by the current through the localized conductive paths, Fig. 1 (b). Then, optimization of the memory operation conditions can be achieved by controlling energy via tuning amplitude and durations of the programming pulses. This was done by employing the ultra-short (100 ps -10 ns) voltage pulsed technique [2], which allows us to evaluate how synaptic weights are modulated by incoming signals. Performance improvement was assessed using a developed phenomenological model based on statistical MonteCarlo simulations for assessment of the memory update performance over many programming events under different operating conditions. This model implements inter-CNT contact properties extracted from DFT simulations of possible CNT-CNT structures.

\section{RESULTS AND DISCUSSION}

Structures of the tubes of various chirality and connections between the tubes (Fig. 3a - 3c) were obtained via DFT modeling and electrical current through contacts was calculated with the NEGF method.

Since electron transport along the tubes is of ballistic nature, the current magnitude through the CNT fabric is primarily controlled by the tube-to-tube contacts. Higher conductive state, LRS, is then determined by closer contacts, while HRS is associated with longer inter-tube distances in the contact region. Change of conductance (switching) is caused by changing contact distance, Fig. 3d, driven by energy and elastic forces inducing tube bending. Repeatable switching can be understood by assuming the existence of stable HRS and LRS inter-tube configurations. However, the calculations show that HRS cannot be stabilized by van der Waals forces, Fig. 4. We proposed a model where HRS is stabilized by a nearby CNT, widely available in the dense random multi-layer film, Fig. 5, that is consistent with the observed abrupt and gradual current time dependencies during Reset and Set pulse operations at constant voltages, respectively. DFT calculations demonstrate that adjacent CNTs can form double-minimum energy state as required for LRS-HRS formation, Fig. 6. Within the double-well model, HRS is expected to be less thermally stable since heating (vibrational excitations) can easier activate a transition over a lower barrier of the higher energy metastable HRS to lower energy LRS. Indeed, such spontaneous temperature-induced transition in a number (but not all) of contacts was observed after a cell was kept at higher $\mathrm{T}$ for $2500 \mathrm{sec}$, Fig. 7.

Based on the DFT modeling results, the phenomenological description (PD) of the device operations was developed to reproduce memory states cycling at the memristor cell level. Initiating (forming) operation leads to formation of a large number of preferential conductive paths (PCP) through the CNT fabric distributed over the device area; each path represents a random chain of contacting CNTs linking top and bottom electrodes, as schematically shown in Fig.8. The total current through the cell is simulated taking into account the distribution of electric field and local temperature (calculated via Joule heating and calibrated based on the ultra-short pulse measurements).

PD simulations data match well to measurements of the current time dependency in nanosecond range, Fig. 9, as well as reflect on I-V nonlinearity in HRS/HRS, Fig. 10. Simulations successfully reproduces measured cell cycling, Fig. 11, and identified structural features responsible for switching variability, Fig. 12. It suggests that engineering a smaller share of wall-towall CNT contacts (see Fig. 3, 12) results in both lower read current (less of interconnection issues in large cell areas) and tighter distribution of conductance values in both low and high resistive states.

\section{SUMMARY}

Ultra-short pulse durations on the timescale of the characteristic switching processes induce small controllable parameters changes thus allowing, in combination with atomiclevel material modeling, to separate different factors affecting switching operations (e.g., released energy, electric field, temperature, tubes chemical functionalization and alignment etc.) and identify structural (modifiable) features and operational conditions leading to improved characteristics of the CNT cell.

[1] D. C. Gilmer et al, "NRAM: a disruptive carbon-nanotube resistancechange memory", Nanotechnology 29 (2018) 134003

[2] D. Veksler, G. Bersuker, et al, "Switching variability factors in compliance-free metal oxide RRAM”, 2019 IEEE International Reliability Physics Symposium (IRPS). 
(a)

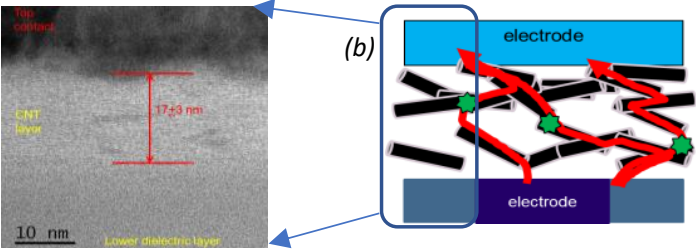

Figure 1. (a) TEM image and (b) schematic of NRAM cell structure with conductive paths (red lines)

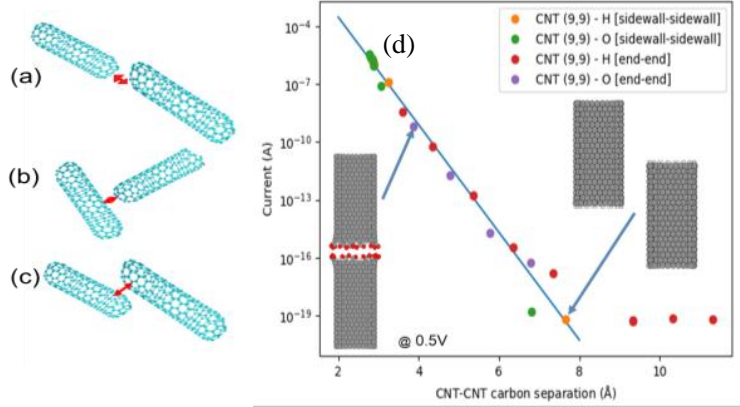

Figure 3. Possible tubes configurations in random CNTs network: (a) tip-to-tip, (b) tip-to -wall, (c) wall-to-wall. (d) Resulted currents, at $V=100 \mathrm{mV}$ and $T=25^{\circ}$ $C$, through the connecting CNTs of $(9,9)$ chirality vs. distance between CNTs in corresponding configurations. Oxygen (red dots) and Hydrogen (open dots) termination at the CNT tips imployed in film fabrication is considered.

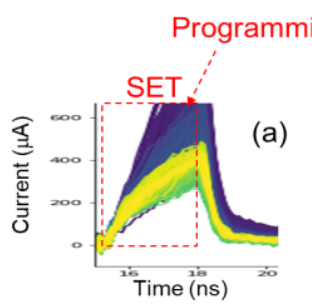

(b)
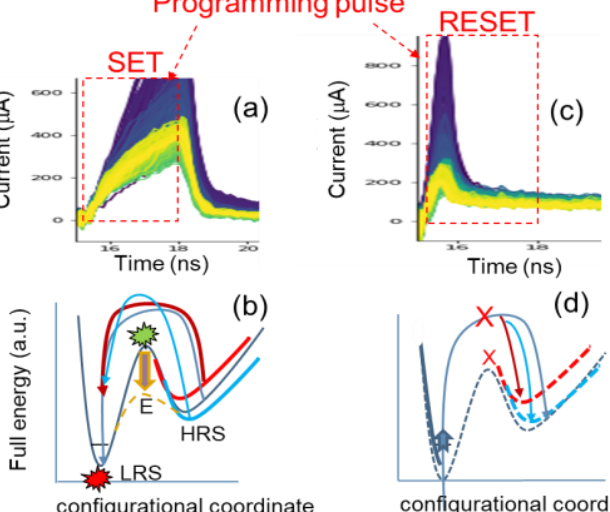

(d)

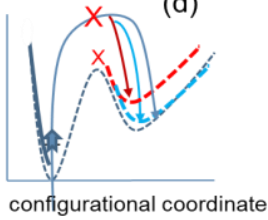

Figure 5. Within a frame of the double-well model: Forming establishes initial set of PCPs with widely distributed HRS contacts; (a-b) Set: energy release enables transitions to lowest v.d. Waals energy contacts between CNTS. (a) SET is gradual due to wide energy minima distribution of post-forming HRS contacts states. (c-d) RESET is driven by returning to the energy-distance distribution of initial post-forming contacts. (c) RESET is abrupt due to similar minimal-energy v.d. Waals arrangmnent in all LRS contacts.

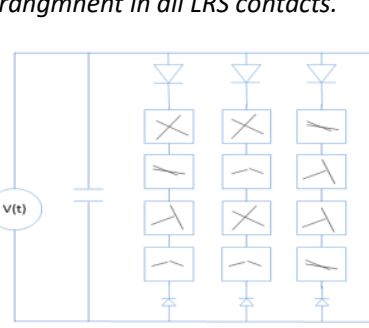

Time dependent

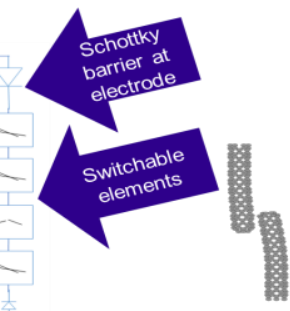

Time independent

describes switching operations $\mid$ describes read measurements under specific pulse duration during cycling

Figure 8. Equivalent circuit model combining individual CNTCNT contacts in a conductive path. Each block is a schematic representation of a possible inter-tubes allignments resulting in a specific contact. Interface between CNT and electrodes is described by a Schottky diode. Programming operations are governed by Poisson sampling to switch individual elements. Total current calculated using Newton-Raphson root finding method for system of nonlinear equations.

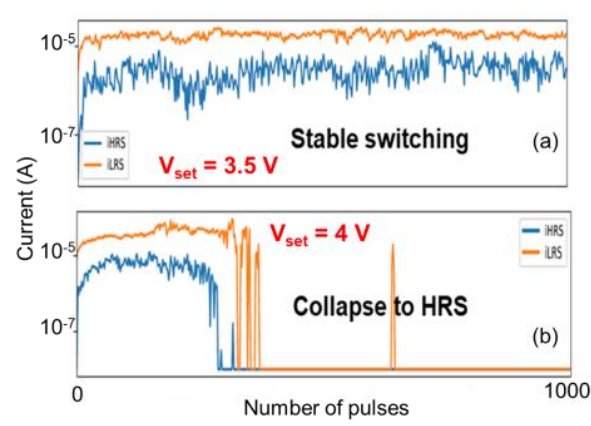

Figure 4. Modelled dependence of inter-CNT energy and contact current controlled by ellastic and van der Waals forces vs. inter-CNTs spacing.

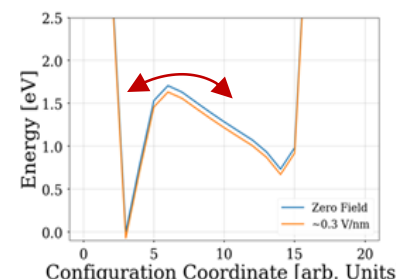

Figure 6. v.d. Waals energy dependency of connecting tubes (\#1 and \#2) in the conductive path vs. inter-tube seperation associated with the switching between LRS ( $x=3 A$ distance) and HRS $(x=14 A)$. Presence of the third tube \#3 stabilises HRS minimum. Electric field induced by the applied voltage

perperdicular to the tubes directions doesn't affect switching characteristics.
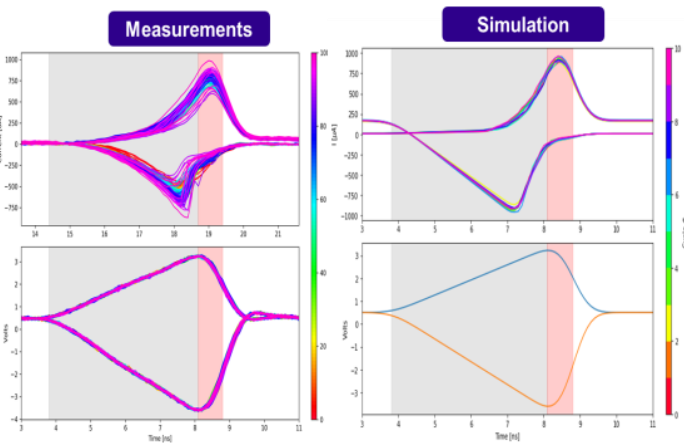

Figure 9. Measurements of Set and Reset currents during 5 nsec repeated switching process and corresponding simulation data.
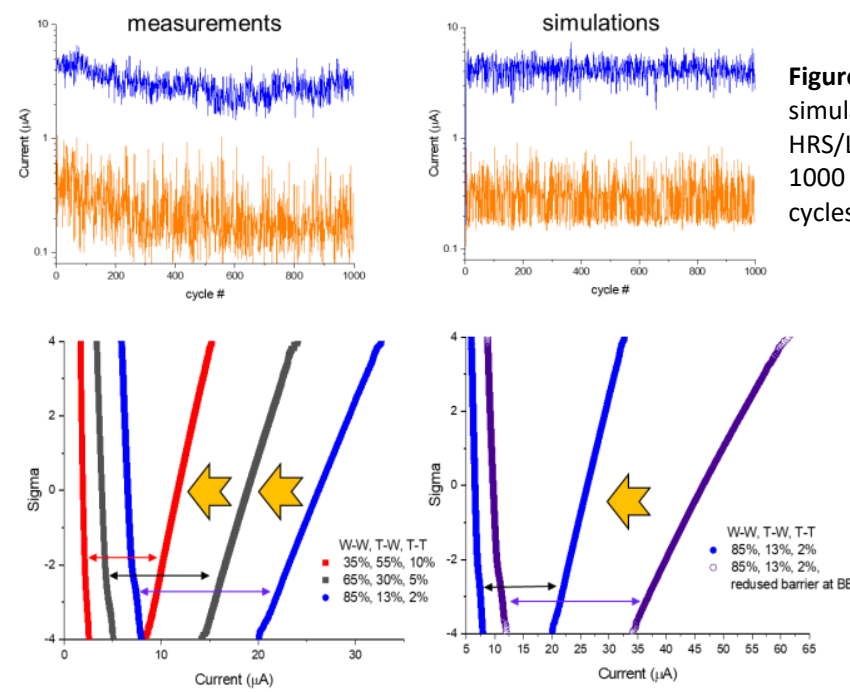

Figure 2. 1000 consecutive bi-polar switching between high (HRS) and low (LRS) resistance states measured under programming pulses of (a) higher $V_{0}+0.25 \mathrm{~V}$ and (b) lower $V_{0}-0.25 \mathrm{~V}$ voltages. Slightly higher operational voltage results in HRS degradation and eventual collapse.
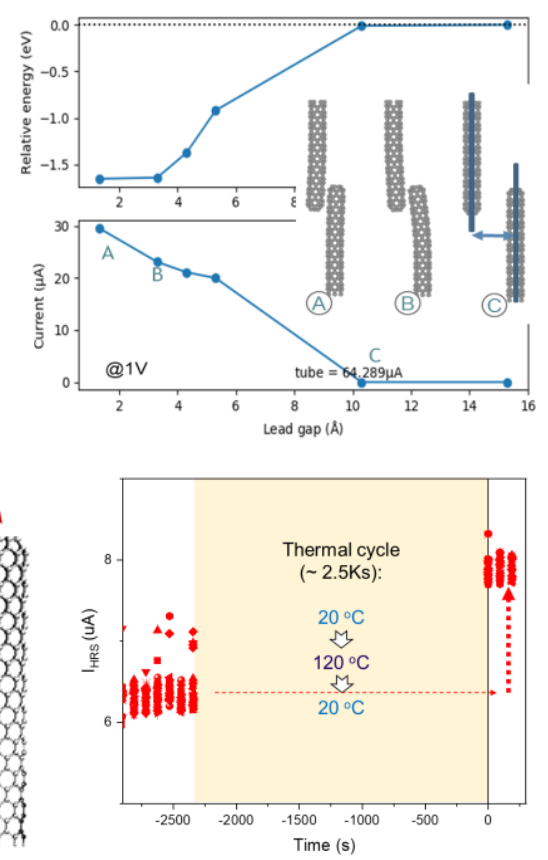

Figure 7. HRS read measurments before and after $120 \mathrm{C}$ heat exposure. HRS conductance increased shifting towards LRS in agreement with the double-well model prediction.

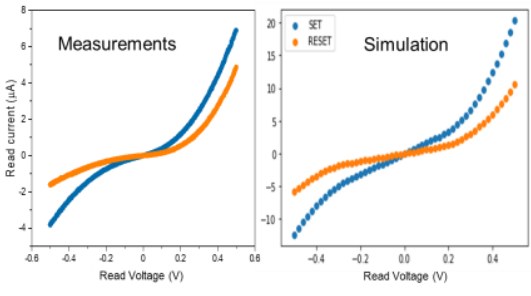

Figure 10. Measured and simulated I-V characteristics in Low and High resistive states

Figure 11. Measured and simulated distributions of HRS/LRS read currents in 1000 set/reset switching cycles.

Figure 12. Simulated sigma-plots for low and high resiatnce state in million switching cycles. Higher share of tip-to-tip (T-T) connections, and greater electrode-CNT barrier are promissing for tightening the conductance distributions and lowering cell read current. 